\title{
Anti-PD1 in the wonder-gut-land
}

\author{
Cell Research (2018) 28:263-264. doi:10.1038/cr.2018.12; published online 16 January 2018
}

\begin{abstract}
After the initial success of cancer immunotherapy using immune checkpoint blockers, the challenge is to understand why only a minority of patients respond to the therapy and to increase the rate of response. Three recent papers now report that the gut microbiota modulates the response to anti-PD1 therapy in patients with epithelial cancers or melanoma.

While the tumor mutational load has been associated with therapeutic response to immune checkpoint blockers (ICBs) [1], the role played by host factors recently emerged. The ability of gut microbiota to modulate the response to cancer chemotherapy and immunotherapy was first described in mice [2-5]. Now, three papers published in Science analyze cohorts of patients and clearly establish the role of gut bacteria in modulating the response to anti-PD1 (Figure 1) [7-9].

Zitvogel's group (Institut Gustave
\end{abstract} Roussy, Villejuif, France), using large cohorts of advanced lung, renal and urothelial cancer patients treated with anti-PD1 in both Europe and USA, established that patients that received antibiotics before or soon after antiPD1 relapse sooner and have an overall survival that was less than half than patients that did not receive antibiotics [7]. Analysis of the composition of 100 lung and renal cancer patient gut microbiota by shotgun metagenomics sequencing revealed that anti-PD1 responders ( $\mathrm{R}$, as defined by either the best response according to Recist1.1 criteria or progression-free disease for 3 months) harbor significantly increased representation of the bacterial species Akkermansia muciniphila than non-responder (NR).
Culture of fecal sample from lung cancer patients also highlighted a higher frequency of Staphylococcus haemolyticus and Corynebacterium aurimucosum in NR patients and a trend for a higher representation of Enterococcus hirae in R patients. Corroborating these studies, memory Th1 and Tc1 responses against $E$. hirae or A. muciniphila were associated with favorable clinical outcome. Furthermore, transfer of the patients' fecal microbiota into germ-free or antibiotics-treated mice replicated the donor ability to respond to ICBs. Administration by gavage of A. muciniphila alone or combined with $E$. hirae rescued the unresponsiveness to anti-PD1 of mice recolonized with fecal microbiome from NR patients. The adjuvant effect of $A$. muciniphila on anti-PD1 response required IL-12 production, as it was previously shown for the effect of Bacteroides fragilis on anti-CTLA4 therapy [4], and induced an increase of gut-tropic $\mathrm{CD} 4^{+} \mathrm{T}$ cells expressing the chemokine receptor CCR9 in mesenteric lymph nodes, tumor beds and tumor-draining lymph nodes.

Wargo's group (MD Anderson Cancer Center, Houston, Texas, USA) explored the role of oral and gut microbiome in anti-PD1-treated melanoma patients by $16 \mathrm{~S}$ rRNA gene sequencing [8]. The oral microbiome did not reveal differences between $R$ and NR patients. However, the analysis of 43 patients' fecal samples, showed that the alpha diversity was significantly higher in $\mathrm{R}$ patients (Recist1.1 response or stable disease at 6 months) with an enrichment of Clostridiales, Ruminococcaceae and Faecalibacterium in R and Bacteroidales in NR patients. Shotgun metage- nomic on 25 samples from the same cohort confirmed the enrichment of Feacalibacterium species in $\mathrm{R}$ patients. Patients with a higher abundance of Faecalibacterium experienced a significantly prolonged progression-free survival, whereas the relative abundance of Bacteroidales was associated with increased risk of relapse. Patients with a favorable composition of gut microbiota at treatment baseline had higher cytotoxic $\mathrm{CD}^{+} \mathrm{T}$ cell infiltration in the tumor bed and evidence of preexisting anti-cancer immune responses. Patients' fecal microbiota transfer into germfree mice showed that a transplantable syngeneic melanoma grew slower, was infiltrated with a higher number of $\mathrm{CD} 8^{+}$ $\mathrm{T}$ cells and responded better to anti-PDL1 therapy in the mice with the fecal microbiota of $\mathrm{R}$ patients than in those with that of NR patients.

Gajewski's group (University of Chicago, IL, USA) reported the analysis of 38 fecal pre-treatment samples from metastatic melanoma patients treated with anti-PD1 [9]. This analysis, based on $16 \mathrm{~S}$ rRNA sequencing, shotgun metagenomic and quantitative PCR, identified many bacterial species that predicted a favorable response to the therapy (as analyzed by Recist1.1 criteria). In particular Bifidobacterium longum (validating previous murine data reported by this group [5]), $\mathrm{Co}$ linsella aerofaciens and Enterococcus faecium were associated with better prognosis. Transfer of patients' fecal microbiota into germ-free mice indicated that, despite heterogeneity in the colonization of human bacterial commensals in germ-free mice, improved tumor control and response to anti-PD- 


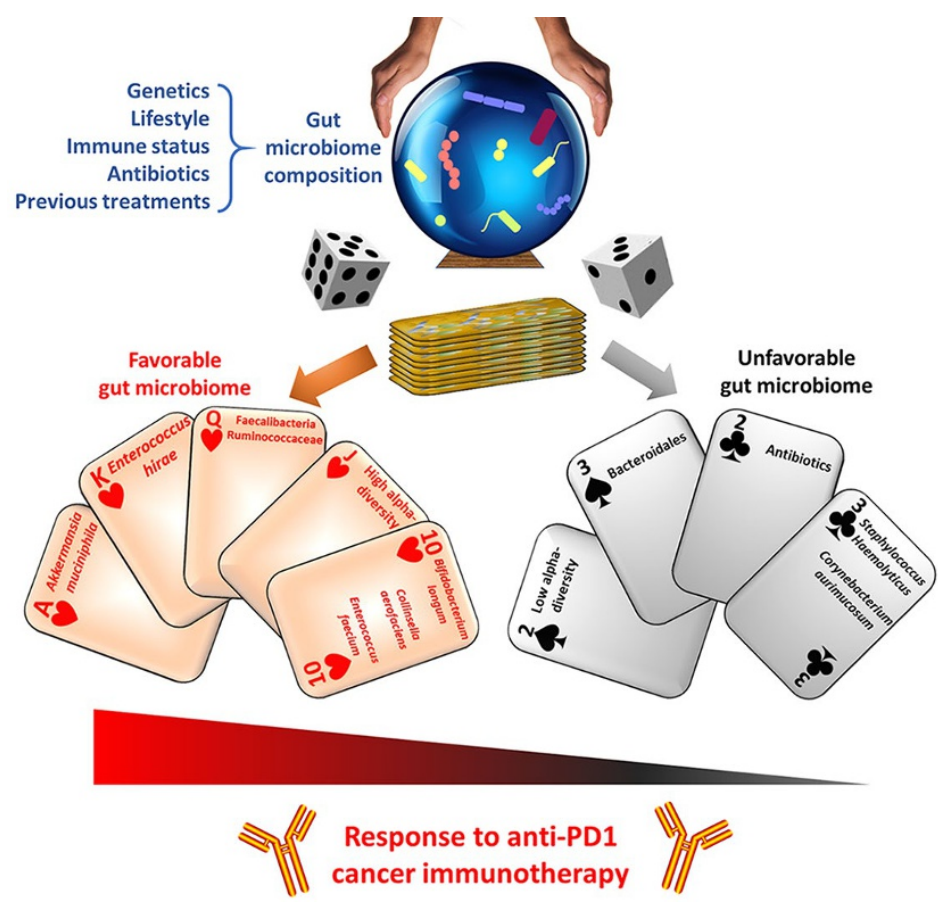

Figure 1 The composition of the fecal microbiota is regulated by the genetics, lifestyle and treatment history of the cancer patients. Three recent papers show that the gut microbiota modulates the ability of the patients to respond to anti-PD1 immunotherapy, may be used as an independent biomarker to predict responsiveness and in the future may be possibly targeted to improve the proportion of patients able to respond.

L1 was observed in mice receiving fecal samples from some $\mathrm{R}$ patients.

Together, these studies provide a clinical demonstration that the gut microbiota modulates the response to inhibitors of PD1-PDL1 axis. Overall, they indicate that a healthier, highly diverse microbiota and the presence of certain bacterial species favor the establishment of an anti-tumor immune response at baseline that may be enhanced by the anti-PD1 treatment with favorable clinical response. Alteration of microbiota balance by antibiotics treatment near the initiation of the therapy reduces its efficacy. However, while all three studies reported the importance of gut microbiota, they highlighted different bacterial players. Many factors impact human microbiota composition, such as diet, drugs, early childhood exposure, stress, and the long-lasting perturbation induced by antibiotics [10]. Thus, the discrepancy may in part to be attributed to the small patient cohorts in geographically distant populations and different criteria for therapy response utilized in these studies. Also, the accuracy of taxonomic identification by shotgun metagenomics is limited by incomplete databases of full bacterial genomic sequences and requires the adoption of new pipelines that are being developed for deeper analysis and assembly that together with culture of clinical bacterial isolates will allow the identification of new species and discrimination between strains of the same species. In addition, the effects of the microbiota on therapy are unlikely to be due to single species but rather to changes in the ecology and metabolism of the gut microbiota that together affect cancer immunity. The identified species or group of species are likely biomarkers of these more complex ecological changes and, because of the small-sized and heterogeneous cohorts analyzed, different species may have reached significance in the three studies. Large clinical studies correcting the population difference and improved bioinformatics analysis will lead to a more precise identification of the composition and metabolic characteristics of the microbiota needed for favorable clinical responses. Then, the challenge will be to target the microbiota to increase the number of patients that can benefit from the therapy. If the progress in the analysis of the microbiota by next-generation sequencing has been at lightning speed, not the same can be claimed for our ability to target the microbiota composition. Still, rudimental methods such as fecal transfer, probiotics and prebiotics are utilized and the use of different formulations of defined bacterial preparations is only slowly developing. Yet, the possibility of targeting the microbiota to improve cancer treatment is an exciting one that is likely soon to become a reality.

\section{Marie Vetizou ${ }^{1}$, Giorgio Trinchieri ${ }^{1}$}

${ }^{I}$ Cancer and Inflammation Program, Center for Cancer Research, National Cancer Institute, National Institutes of Health, Bethesda, MD 20892, USA

Correspondence: Giorgio Trinchieri

E-mail: trinchig@niaid.nih.gov

\section{References}

1 Rizvi NA, Hellman MD, Snyder A, et al. Science 2015; 348:124-128.

2 Viaud S, Saccheri F, Mignot G, et al. Science 2013; 342:971-976.

3 Iida N, Dzutsev A, Stewart CA, et al. Science 2013; 342:967-970.

4 Vétizou M, Pitt JM, Daillère R, et al. Science $2015 ;$ 350:1079-1084.

5 Sivan A, Corrales L, Hubert N, et al. Science 2015; 350:1084-1089.

6 Rosshart SP, Vassallo BG, Angeletti D, et al. Cell 2017; 171:1015-1028.

7 Routy B, Le Chatelier E, Derosa L, et al. Science 2018; 359:91-97.

8 Gopalakrishnan V, Spencer CN, Nezi L, et al. Science 2018; 359:97-103.

9 Matson V, Fessler J, Bao R, et al. Science 2018; 359:104-108.

10 Namasivayam S, Maiga M, Yuan W, et al. Microbiome 2017; 5:71. 